\title{
Clinical Characteristics of Cervicogenic-Related Dizziness and Vertigo
}

\author{
Dario A. Yacovino, $M D^{1}$ Timothy C. Hain, MD $^{2}$ \\ 1 Section of Neuro-otology, Neurology research institute “Dr, Raúl \\ Carrea" (FLENI), Buenos Aires, Argentina \\ ${ }^{2}$ Departments of Neurology, Otolaryngology, Physical therapy and \\ Human Movement Science, Northwestern University, Chicago, \\ Illinois
}

\begin{abstract}
Address for correspondence Dario A. Yacovino, MD, Section of Neurootology, Neurology research institute "Dr, Raúl Carrea" (FLENI), Montañeses 2325 (C1428QK), Ciudad autónoma de Buenos Aires, Argentina (e-mail: yac@intramed.net).
\end{abstract}

Semin Neurol 2013;33:244-255.

\begin{abstract}
Keywords

- vestibular function

- vestibular recovery

- vertigo

- cervical vertigo

- dizziness

- vertebrobasilar insufficiency

Cervical vertigo has long been a controversial entity and its very existence as a medical entity has advocates and opponents. Supporters of cervical vertigo claim that its actual prevalence is underestimated due to the overestimation of other diagnostic categories in clinics. Furthermore, different pathophysiological mechanisms have been attributed to cervical vertigo. Here the authors discuss the clinical characteristics of rotational vertebral artery vertigo, postwhiplash vertigo, proprioceptive cervical vertigo, and cervicogenic vertigo of old age. A clinical entity named subclinical vertebrobasilar insufficiency appears in the context of cervical osteoarticular changes. Migraineassociated vertigo may explain why some patients suffering from cervical pain have vertigo while others do not.
\end{abstract}

\section{General Background}

Vertigo and dizziness are among the 20 most common causes of consultation in adult patients. ${ }^{1}$ In $80 \%$ of these cases, the symptoms are so intense as to require medical intervention. Dizziness affects more than $50 \%$ of the population over 65 and is the most frequent reason for medical consultation after the age of $75 .{ }^{1}$ Roughly $50 \%$ of patients who are referred for dizziness have vertigo. Among the various causes of vertigo, the so-called cervical vertigo, basically vertigo caused by neck disorders, has been the most controversial. Neurologists often face the situation of having to confirm or reject a diagnosis given by other specialists. Yet, the existence of cervical vertigo is not questioned in physiotherapy settings, possibly due to the extensive bibliography covering this condition in this specialty. ${ }^{2}$

Cervical vertigo can be a rare cause of vertigo ${ }^{3}$ or it can be among its main causes. ${ }^{4}$ In the elderly, Colledge et al suggested that cervical spondylosis is the second most-frequent cause of dizziness, which would be more representative of those who complain of dizziness to their general practitioner. $^{5}$

Advocates of vertigo of cervical origin state that cervical vertigo is the most common cause of vertigo; however, there is no diagnostic method to establish whether a patient's vertigo is caused by an underlying neck condition. The central cervical vertigo hypothesis-that a neck condition causes dizziness-is plausible because dizziness is associated with head and cervical spine rotation. This could have possibly perpetuated the concept of cervical vertigo in nonmedical forums. Nevertheless, due to the lack of a specific diagnostic test and the condition's overlap with other commonly diagnosed conditions that also have no specific tests, cervical vertigo is still a controversial entity: Many patients preliminarily diagnosed with such a disorder are ultimately found to have other pathologies. ${ }^{6}$

Our first objective in this review is to evaluate extensively and critically the scientific bibliography in search of the historical origin of cervical vertigo and its clinical variants. Our second objective is to analyze the anatomy and function of the neck in relation to cervical vertigo and its clinical presentation. Finally, we formulate an alternative hypothesis about the symptoms that are attributed to this condition.

\section{Neck Structures}

The neck has structures that are involved in

1. Balance control (cervical afferents)
Issue Theme Neuro-Otology 2013; Guest Editor, Terry D. Fife, MD
Copyright $\odot 2013$ by Thieme Medical Publishers, Inc., 333 Seventh Avenue, New York, NY 10001, USA. Tel: +1(212) 584-4662.
DOI http://dx.doi.org/ 10.1055/s-0033-1354592. ISSN 0271-8235. 
2. Vascular tone control (carotid sinuses)

3. Blood circulation (carotid and vertebral arteries)

4. Support of the cervical spinal cord

Movements of the head invariably include cervical movements; therefore, symptoms such as dizziness, imbalance, or vertigo associated with cervicocephalic movements could also be attributed to a vestibular (inner ear), visual, vascular, neurovascular, cervicoproprioceptive, or cervical spinal cord dysfunction.

\section{Cervicovestibular Integration}

The vestibular apparatus of the inner ear (semicircular canals and otolith; "Vestibular" in - Fig. 1) detects the velocity, acceleration, and indirectly the position (e.g., gravity) of the head. Other motion inputs to the vestibular system include position sensation and especially cervical proprioceptive afferents, visual signals, and intended movement (efference copy) including cerebellar projections of the Purkinje cells ${ }^{7}$ ("Motor commands" in - Fig. 1). These redundant inputs are integrated by the central processor, the vestibular nuclear complex, which generates motor commands to drive the eyes and body. The system is normally very accurate. To maintain accuracy, it is monitored and calibrated by the cerebellum. The integrated perception of head rotation in space can be disturbed and inaccurate if the input streams do not agree.

Somatosensory signals by themselves can generate vertigo. The cervico-ocular reflex-eye movement developed purely from neck torsion-has been well documented in humans, and can be very substantial in certain populations with diminished vestibular input. ${ }^{8,9}$ Huygen et $\mathrm{al}^{9}$ documented mean COR gains ranging between 0.41 (at $0.4 \mathrm{~Hz}$ ) and 0.72 (at $0.1 \mathrm{~Hz}$ ) in 30 labyrinthine defective subjects. Furthermore, Brandt et $\mathrm{al}^{10}$ showed that it is possible to induce nystagmus in the dark by passively rotating an arm (arthrokinetic nystagmus). Additionally, Bles et al ${ }^{11}$ reported that vertigo and nystagmus can be generated in persons without a labyrinth through "stepping around." De Jong et al showed that blocking of cervical afferents without any vestibular stimulation produces nystagmus in a variety of animals. ${ }^{12}$ Another line of evidence that somatosensory input can cause vertigo is that it has been well documented that neck vibration induces nystagmus in unilateral vestibular loss conditions. ${ }^{13}$ It has also been our observation that these patients state that vibration of the sternocleidomastoid often "makes them dizzy." Similarly, in studies conducted in humans using cervical affects such as endogenous (pain), ${ }^{14}$ external chemical agents (local anesthesia), ${ }^{12,15}$ and physical (motion, ${ }^{16}$ vibration, ${ }^{17,18}$ sustained rotation ${ }^{19}$ ) and galvanic stimulation, ${ }^{20}$ it was possible to cause postural imbalance, pulsion, subjective displacement of verticality, nystagmus, and vertigo albeit rarely.

In summary, to maintain balance and generate appropriate motor responses for equilibrium, signals from the eyes (vision), the ear (vestibular system), proprioception (spatial orientation of the body segment), and internal estimates and efference copy signals are combined. Furthermore, proprioceptive signals from the neck can generate nystagmus and vertigo.

\section{Cervical Vertigo}

Cervical vertigo shares the term "vertigo" with height vertigo, phobic vertigo, and some forms of visual vertigo. However, the symptomatology described in articles about cervical vertigo does not usually include an illusory perception of rotational or linear movement that defines vertigo, but rather fits the definition of imbalance (reduced ability to keep or control balance), or more broadly, the definition of dizziness (unpleasant and vague feeling that involves the individual and the environment, unsteadiness, spatial discomfort, or dullness). In this context, the term "cervical vertigo" is tacitly accepted. We will try to clarify such ambiguity throughout the text.

In summary, when it comes to define cervical vertigo either as a disease or as a clinicopathological entity, the following problems arise:

- The definition of cervical vertigo is neither accurate nor uniform.

- There are neither epidemiological data nor populationbased studies (the only exception is postwhiplash vertigo).

- There are no specific tests for cervical vertigo.

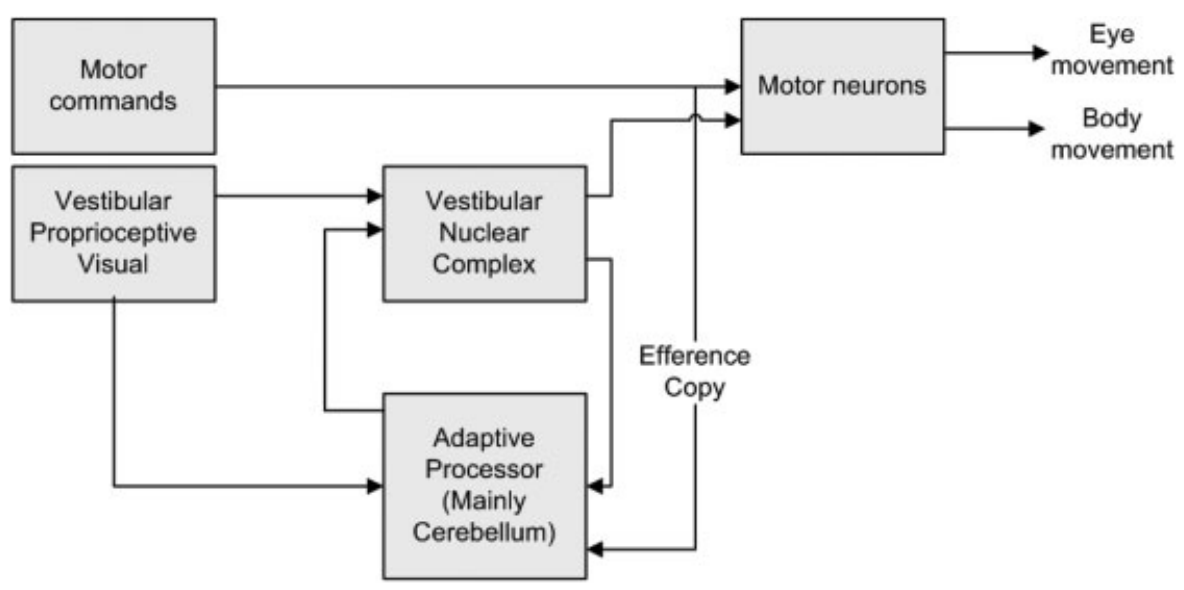

Fig. 1 Somatosensory integration. The vestibular system integrates motor commands (efference copy) with vestibular, proprioceptive, and visual inputs. 
- There are no pathognomonic clinical elements.

- There is no clear pathophysiological support.

- If appropriate diagnostic methods are used, the symptoms have other possible causes in $90 \%$ of the cases. ${ }^{21}$

Based on the above, considerable effort must be made to classify cervical vertigo as a disease. Nevertheless, there are several publications reporting efficacy of physical therapy alone or in association with drugs for the treatment of cervical vertigo. ${ }^{2,22-24}$

Furman and Cass ${ }^{25}$ offered a definition for cervical vertigo. They used the expression "cervicogenic dizziness" and defined it as a vague feeling of balance alteration and spatial orientation that is produced by an abnormal activity of the cervical afferents. The authors postulated that cervicogenic dizziness is not the result of a vestibular dysfunction; therefore, it is unlikely to cause true vertigo. However, as noted in the literature review above, there is very good evidence that purely proprioceptive signals can generate nystagmus and vertigo. Furthermore, this definition has no utility for diagnosis.

Yahia et $\mathrm{al}^{26}$ defined cervical vertigo as an association of the following features: chronic cervical pain, vertigo after cervical rotation without nystagmus, cervical osteoarthritis, and/or intervertebral disk degenerative changes. This definition, depending primarily on a subjective sensation and a collection of common clinical complaints, is too inclusive to be generally useful.

According to Wrisley, the diagnosis of cervicogenic dizziness is dependent upon correlating symptoms of imbalance and dizziness with neck pain and excluding other vestibular disorders based on history, examination, and vestibular function tests. ${ }^{27}$ Although vague and lacking the inclusion of proprioceptive loss as experimental studies have estab- lished, ${ }^{12}$ this definition is superior for clinical purposes to that of Yahia et al as it requires exclusion of alternatives. However, by allowing diagnoses made by clinical pattern matching, such as migraine-associated vertigo or chronic subjective vertigo to exclude the diagnosis of cervical vertigo, it could allow for considerable variability in diagnostic processes.

\section{Hypotheses Historically Supporting Cervical Vertigo}

According to Heikkila, ${ }^{28}$ since the beginning of the last century, three different hypotheses explaining the vertigo that originates in the cervical area have been put forward. These three theories are summarized in -Table 1.

The Neurovascular Hypothesis: The Barré-Lieou Syndrome The first of the theories concerning the mechanism for cervical vertigo was proposed in 1928 by Jean-Alexander Barré and Young-Choen Lieou. ${ }^{29}$ They proposed that the sympathetic plexus surrounding the vertebral arteries could be mechanically irritated by degenerative changes in the cervical spine and the sympathetic irritation could produce reflexive vasoconstriction in the vertebrobasilar system, thus accounting for the symptoms of disequilibrium. Experimental data showed that sympathetic denervation does not cause vasodilatation in normotensive animals, nor does irritation produce vasoconstriction. ${ }^{30,31}$ The Barré-Lieou syndrome, thus, was discredited. ${ }^{32}$ Still, references to the Barré-Lieou syndrome are found in journals concerning chiropractic or legal medicine.

\section{The Somatosensory Input Hypothesis}

Around 1955, Ryan and Cope ${ }^{33}$ coined the term "cervical vertigo." They were the first to propose the somatosensoryinput pathophysiology of cervical vertigo. They suggested that its origin stemmed from the existence of abnormal

Table 1 Schematic overview of three commonly proposed mechanisms for cervical vertigo

\begin{tabular}{|l|l|l|}
\hline Denomination & Pathophysiological background & Review \\
\hline $\begin{array}{l}\text { Barré-Lieou syndrome (sympathetic } \\
\text { cervical syndrome) }\end{array}$ & $\begin{array}{l}\text { Posterior circulation, including the blood } \\
\text { vessels of the inner ear, is innervated by a } \\
\text { sympathetic system from the cervical nerve } \\
\text { roots. The compression of these roots could } \\
\text { produce irritation of the postganglionic } \\
\text { sympathetic system and generate } \\
\text { vasoconstriction and inner-ear ischemia. }\end{array}$ & There are no conclusive studies. \\
\hline Proprioceptive cervical vertigo & $\begin{array}{l}\text { Cervical proprioception integrates in the } \\
\text { vestibular nuclei, exerts a modulating effect } \\
\text { (vestibulospinal path), and facilitates or inhibits } \\
\text { the peripheral vestibular discharges (the richer } \\
\text { zones in receptors are joint capsule C1-C2, } \\
\text { suboccipital muscles, and deep paravertebral } \\
\text { muscles) }\end{array}$ & $\begin{array}{l}\text { According to current evidence, these } \\
\text { connections would be unlikely to }\end{array}$ \\
\hline $\begin{array}{l}\text { Vertigo in rotational vertebral artery } \\
\text { syndrome (bow-hunter syndrome) }\end{array}$ & $\begin{array}{l}\text { Studies performed in cadavers showed that } \\
\text { head rotation toward one side compresses the } \\
\text { vertebral artery of the opposite side at the atlas } \\
\text { and axis level and produces vertebrobasilar } \\
\text { insufficiency. }\end{array}$ & $\begin{array}{l}\text { It is possible, although it requires } \\
\text { several anatomical alterations that } \\
\text { makes it infrequent. }\end{array}$ \\
\hline
\end{tabular}


sensory information coming from the damaged joint receptors of the upper cervical regions. Such irregular and asymmetric information could alter the vestibular nuclei of the brainstem as reviewed above.

Although Ryan and Cope offered a viable hypothesis for the mechanism of cervical vertigo, on analysis of the medical histories of the five patients described in their original article, four of them showed symptoms that were more consistent with paroxysmal positional vertigo accompanied by cervical radicular involvement. They may have been unaware that just 3 years before in London, Dix and Hallpike had described 100 patients who had like symptoms and named this clinical condition "benign paroxysmal positional vertigo" (BPPV). ${ }^{34}$

In addition, Brown added the concept that spasm or contracture of the sternocleidomastoid and upper trapezius muscles might generate erratic muscular-sensory information. ${ }^{35}$ Morinaka and associates suggested that the interconnection between cervical proprioception and vestibular nuclei can create a cyclical pattern in which imbalance could produce cervical muscle contracture and spasm, and in turn contribute to an increase in symptoms. ${ }^{36}$ However, it is difficult to show in an experimental fashion this relation. To summarize, at the present state of knowledge, the theory that cervical nystagmus and vertigo are caused by an alteration of the function of somatic neck afferents in humans is supported by strong indirect evidence.

There are several epidemiological subgroups of cervical vertigo, for which the somatosensory mechanism is thought to apply. Dizziness in combination with cervical disorders is a common complaint in older patients. In this group, dizziness consists of defects in orientation, unsteadiness, but rarely true vertigo. This age group often also suffers from vascular, osteoarticular, metabolic, and sensory degenerative problems. The elderly also tend to take many medications. All of these conditions can cause dizziness and/or vertigo. In a community-based controlled study, ${ }^{5}$ osteoarticular changes were attributed to be the second most common cause of dizziness in the elderly. It is well known that the vestibular and the proprioceptive systems deteriorate in older adults. ${ }^{37,38}$ When cervical afferents are altered by neck osteoarticular or muscle (pain) changes, the nervous system attempts to reweight sensory information to the vestibular system. ${ }^{38,39}$ Due to the deterioration of the vestibular system in older adults, there is no other immediate, reliable, sensory source to trust. Within this new sensory framework, the head position sense is impaired and this results in dizziness and/or disorientation. This type of cervicogenic dizziness is a diagnosis of exclusion and has no distinguishing features from "unknown cause" vertigo or multisensory disequilibrium of the elderly.

Another subgroup of cervical vertigo, generally attributed to somatosensory disturbance, is whiplash-associated disorder. Whiplash-associated disorder is worthy of a comment due to its high prevalence. Traffic accidents accompanied by a rear-end collision are the most frequent cause of whiplashassociated disorder. The general adoption of mechanisms of thorax fixation, such as the seat belt and shoulder straps, considerably reduced serious body injuries from traffic acci- dents, but increased the prevalence of cervical injuries. When the thorax is fixed, the unfixed head and cervical spine absorbs the energy by means of acceleration and deceleration and the consequences can be significant. Whiplash-associated disorder is a polymorphic picture with a complex relation among symptoms due to physical, psychological, and legal factors.

The most common and constant symptoms of whiplashassociated disorder are craniocervical and shoulder pain. Twenty-five to $50 \%$ of patients with whiplash-associated disorder experience vertigo and dizziness; 20 to $25 \%$ of these cases are positional. ${ }^{40,41}$ The chronic variant of whiplashassociated disorder ( $>6$ months) called "chronic whiplash syndrome" is often questioned as legal and psychological interactions may increase the likelihood of protracted symptoms. ${ }^{42}$

Vertigo is not the most common symptom reported by patients with whiplash-associated disorder (except those who have an associated vestibular involvement). On the contrary, sensations of light/heavy/full headedness as well as imbalance are the most commonly encountered. Multiple somewhat tenuous test abnormalities have been reported. Rubin et al ${ }^{43}$ compared posturography in 29 patients with whiplash to 51 healthy subjects, and reported significantly greater abnormalities in the balance of the patients. Abnormal studies of peripheral vestibular function were documented in 4 to $40 \%$ of the cases. ${ }^{44,45}$

Endo et al claimed that patients with cervical vertigo after whiplash injury had an asymmetric reduction in the vertebral arterial flow that was similar to that observed in the cases of vertebrobasilar insufficiency. ${ }^{46}$ Cases of vertebral artery dissection have been reported after whiplash. ${ }^{47}$ However, the existence of pain, dizziness, and imbalance without clear vertigo or brainstem signs makes the vertebral arterial disease theory unlikely in most patients.

On the contrary, it seems more reasonable that in whiplash-associated disorder, pain, limitation of movement, and strains of the joint capsule, the paravertebral ligaments, and the cervical musculature could modify the proprioceptive cervical balance in a sustained way and produce mild but chronic symptoms; in other words, a proprioceptive cervical mechanism is involved. In short, cervical vertigo after whiplash injury represents a particular, prevalent, and known biological model in which some of these patients could attribute their symptoms (vertigo and imbalance) to vestibular alterations and in some patients, to an organic cervical dysfunction.

\section{The Vascular Hypothesis: Compression of the Vertebral Arteries}

Generically, the syndrome produced by the decreased flow of the vertebrobasilar axis of any origin is called "vertebrobasilar insufficiency" (e.g., atheromatosis, vasculitis, subclavian steal syndrome). In particular, the vertigo caused by the compression of one or both vertebral arteries during cephalic rotation is called "rotational vertebral artery vertigo"; it is also called "bow-hunter syndrome." This syndrome was first described in 1978 by Sorensen. He reported a patient who became 
symptomatic when practicing archery. ${ }^{48}$ This entity, although infrequent, is clinically relevant. The physiological collateral vessels and the redistribution of the flow caused by the circle of Willis serve as collaterals when one of the vascular axes is compromised. This explains why most of the patients who suffer from this syndrome often have other associated vascular anomalies (e.g., relative vertebral hypoplasia, a vertebral artery that does not join the basilar, lack of posterior communicating arteries, etc.) that interfere with the compensatory blood redistribution systems.

The theory of the vascular compression arose from hemodynamic studies in cadavers performed by Toole and Tucker in 1960 , who determined that head rotation is accompanied by the compression of the vertebral artery opposite to the direction of rotation-a physiological condition. ${ }^{49}$ According to this proposal, compression of one or both vertebral arteries would be accompanied by brainstem ischemia (vestibular nuclei) and would cause other symptoms aside from vertigo. More specifically, Grad and Baloh ${ }^{50}$ reported that, out of 49 patients with vertigo affecting the vertebrobasilar system, 29 had visual disorders (diplopia, visual field defects, etc.), 14 suffered drop attacks, and nine experienced ataxia and imbalance. This means that this set of symptoms could be technically defined as episodes of transient ischemic attack (TIA) with the risk of morbidity and mortality that entails.

Subsequent studies using digital angiography by Takahashi et al confirmed that the artery compression is neither hemodynamically relevant nor physiological in vivo. ${ }^{51}$ Additionally, after a three-dimensional analysis of the nystagmus in four patients with rotational vertebral artery vertigo, Choi et al concluded that the ischemia and the symptoms were attributed to a vestibular disorder at the ear level instead of at the brainstem level. ${ }^{52}$ This conclusion was supported by the presence of unilateral tinnitus in these patients. Toole and Tucker's theory lost support for this reason. Subsequently, the accurate identification of new cases with different nystagmus helped to highlight the existence of a clinical variability that corresponds to the vascular anatomical variability in the posterior territory (-Fig. 2). ${ }^{53}$

In summary, recurrent episodes of vertigo associated with cephalic rotation can be a consequence of a mechanical vertebrobasilar involvement, although this is not frequent.

\section{Symptomatology}

In proprioceptive cervical vertigo-dysfunction of the joints, muscles, and ligaments-the following symptoms typically occur: pain and limited range of cervical movement, dizziness, imbalance, a motion sickness sensation, aural fullness, phonosensitivity, photosensitivity, and nausea. ${ }^{54}$ In general, the symptoms become more severe with cephalic movements and they can be episodic and last from minutes to hours. As mentioned earlier, this type of mechanism is infrequently associated with true spinning vertigo.

In the case of cervical vertigo after a whiplash injury (whiplash syndrome), the most relevant local complaints among 146 patients were neck pain (98\%), cervical stiffness (95\%), headache (72\%), and scapular pain (20\%). ${ }^{55}$ In 25 to $50 \%$ of the cases, there were complaints of dizziness and vertigo, tinnitus in $14 \%$, and hearing loss in $5 \%$.

In the case of decreased blood flow due to rotational vertebral artery mechanical compression, the symptoms are expected to be essentially the result of the initial ischemic depolarization of the neurons and the inner ear hair cells, which generates irritative symptoms such as tinnitus (unilateral or bilateral), phosphenes, paresthesias, vertigo, and/or nystagmus. If the ischemia persists for approximately 15 or more seconds, lesion phenomena will arise (hearing loss of high-frequency tones, gait instability, vertigo, diplopia, etc.) and pain will be the less relevant symptom. An interesting and unexpected finding was described by Choi et al, ${ }^{52}$ which we have also observed. It was the presence of fatigability or clinical habituation; in other words, when the cervical rotation maneuver is repeated, the induced nystagmus reduces or disappears. Such fatigue, classically attributed to BPPV, could lead to misdiagnoses if head rotation were done supine.

\section{Differential Diagnosis}

The definition of cervical vertigo as the combination of cervical pain and vertigo caused by cephalic rotation as the only diagnostic criterion for cervical vertigo as proposed by Yahia et $\mathrm{al}^{26}$ could generate an overestimation of cervical vertigo. In view of the lack of clear criteria, the most reasonable approach is to consider the diagnosis after ruling out other possible sources of vertigo.

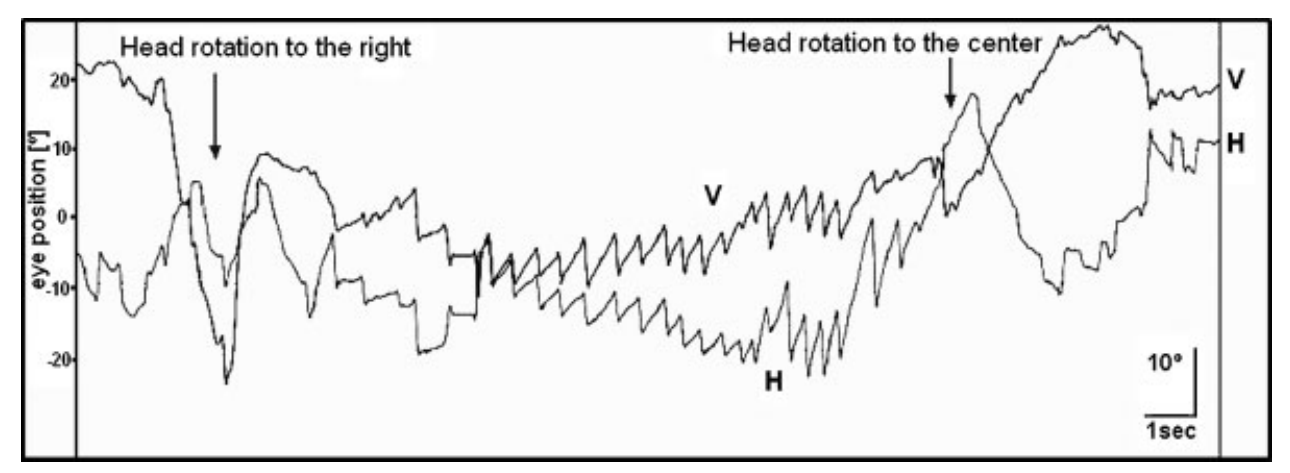

Fig. 2 Recording of spontaneous nystagmus-before and during the passive rotation of the head to the right-of a patient with rotational vertebral arterial syndrome. After a latency of 3 seconds, an oblique nystagmus (left and downbeating) appears and disappears when the original position is restored (looking forward) in a seated patient. The rotation produced a high compression (C1-C2) of the dominant left vertebral arteries. (V, vertical channel; $\mathrm{H}$, horizontal channel). 
- Table 2 lists the most frequent differential diagnoses to cervical vertigo. It is important to highlight BPPV in this group because of its provocation with head turning and high prevalence, BPPV is often misdiagnosed. In BPPV, vertigo develops with head positional changes in space namely, when lying down, rotating in bed, or looking at a shelf that is above us-but it does not happen with relative head rotation with respect to the trunk (when the head is fixed and the trunk is rotated). Positional changes are accompanied by brief and intense vertiginous spells associated to nystagmus from the affected semicircular canal.
Vestibular migraine, a highly prevalent condition, generally combines vertiginous spells, imbalance, intolerance to cephalic movement, and at times, headache and/or neck pain. In this condition, the treatment of migraine is usually effective in more than $80 \%$ of cases. ${ }^{56}$ Although it was not mentioned among the differential diagnoses, in the event of posttraumatic vertigo (e.g., whiplash syndrome), not only are the cervical vertebrae potentially vulnerable to mechanical damage, but also other structures: the ear (labyrinth contusion), the brainstem, the cortical and subcortical structures, and the vertebral arteries (traumatic artery dissection).

Table 2 Clinical syndromes in the differential diagnosis of cervical vertigo with their diagnostic criteria

\begin{tabular}{|c|c|}
\hline Clinical syndrome & Clinical criteria \\
\hline Vertigo & Feeling of movement (oneself or surroundings) \\
\hline Benign paroxysmal positional vertigo & $\begin{array}{l}\text { Vertigo that occurs only with positional changes of the head with respect to } \\
\text { gravity; it usually lasts few seconds. Patients frequently have vertigo and } \\
\text { nystagmus during the positional maneuvers. }\end{array}$ \\
\hline Vestibular neuritis & $\begin{array}{l}\text { Solitary episode of vertigo that is severe at the beginning and usually last } \\
\text { days or weeks. During the chronic phase (uncompensated), dizziness after } \\
\text { head rotation and reactive painful cervical stiffness may appear. }\end{array}$ \\
\hline Ménière’s disease & $\begin{array}{l}\text { Recurrent episodes of vertigo that last a few hours with persistent or } \\
\text { fluctuating hearing impairment, which is recorded through audiometric } \\
\text { tests; characteristic of cases of low-frequency tones, tinnitus, or feeling of } \\
\text { aural fullness. }\end{array}$ \\
\hline Bilateral vestibulopathy & $\begin{array}{l}\text { Imbalance is involved more than vertigo. Physical or neuro-otologic findings } \\
\text { consistent with peripheral bilateral vestibular affection with no involvement } \\
\text { of the central nervous system. }\end{array}$ \\
\hline Central vertigo & $\begin{array}{l}\text { Vertigo not related to benign paroxysmal positional vertigo, vestibular } \\
\text { neuritis, or Ménière's disease, along with the presence of typical central } \\
\text { nystagmus (vertical, rebound, dissociated nystagmus, etc.) during the } \\
\text { oculomotor evaluation or findings consistent with involvement of the } \\
\text { central nervous system in the neurologic exam. }\end{array}$ \\
\hline Vestibular migraine & $\begin{array}{l}\text { Diagnosis of migraine according to ICHD-II criteria, plus the presence of } \\
\text { episodic or fluctuating vestibular symptoms (nonconstant symptoms). } \\
\text { There can be usually reactive cervical pain and cervical trigger points. Other } \\
\text { causes may be dismissed through a neurologic exam and appropriate } \\
\text { studies. }\end{array}$ \\
\hline Vertigo in rotational vertebral arterial syndrome & $\begin{array}{l}\text { Vertigo that does not fulfill the criteria for any of the above-mentioned } \\
\text { types. It is accompanied by signs of vascular dysfunction in the posterior } \\
\text { territory or central nystagmus triggered by head rotation (not positional). }\end{array}$ \\
\hline Vestibular parosmia (neurovascular contact) & $\begin{array}{l}\text { Vertigo of brief duration }(<30 \mathrm{~s}) \text {; it is spontaneous or triggered by head } \\
\text { rotation. It may present brief tinnitus that precedes the attack. Imaging } \\
\text { tests may identify a contact between an artery and the vestibular nerve } \\
\text { (arterial loop) or be normal. }\end{array}$ \\
\hline Chiari malformation type 1 & $\begin{array}{l}\text { It may be accompanied by headache, numbness and cervical stiffness, } \\
\text { imbalance, and positional vertigo. The symptoms usually increase with } \\
\text { physical exercise. }\end{array}$ \\
\hline Imbalance & $\begin{array}{l}\text { Imbalance (usually with sensation of falling) during gait or when standing } \\
\text { still; frequently with no cephalic sensation. }\end{array}$ \\
\hline Multisensory imbalance & $\begin{array}{l}\text { Imbalance and at least two of the following factors: peripheral neuropathy, } \\
\text { visual deterioration, vestibular alterations during the physical examination } \\
\text { or the neuro-otologic exam. It can have a severe reactive cervical stiffness. }\end{array}$ \\
\hline Undetermined imbalance & $\begin{array}{l}\text { Imbalance caused neither by multisensory involvement nor by any other } \\
\text { evident reason. In case of associated neck pain, the diagnosis of proprio- } \\
\text { ceptive cervical vertigo should be considered. }\end{array}$ \\
\hline
\end{tabular}

aCHD-II; Olesen J, Bousser M-J, Diener H-C, et al. International Classification of Headache Disorders (2nd ed.). London, UK: International Headache Society; 2003. 
In contrast, phobic postural vertigo and the so-called somatoform dizziness (or psychiatric vertigo) are differential diagnoses for dizziness in the absence of objective findings.

Patients who suffer from vertigo and imbalance of any origin generally have comorbid cervical muscular tension, stiffness, and pain. ${ }^{57}$ These may be physiological reactions to the perception of less stability and risk of falling. This postural behavior is easily observed and manifested in conditions of instability not only in healthy individuals (e.g., when walking on ice), but also in patients with pathological conditions (e.g., uncompensated vestibular hypofunction). In these cases, the posture of tension and the cervical pain are clearly reactive and improve when balance is restored. Hence, it could be considered an epiphenomenon and not the primary cause of the problem.

\section{Tests for Cervical Vertigo}

We will next discuss special tests for cervical vertigo, laboratory tests for vestibular dysfunction, and imaging studies.

By "special tests," we mean procedures developed, for either bedside or laboratory use, intended to be sensitive and specific for cervical vertigo. An ideal bedside or laboratory test for cervical vertigo would be sensitive and specific, and performed with readily available equipment. As of this writing despite multiple attempts reviewed below, a suitable special test is not available for cervical vertigo.

Some authors have tried to detect the cervical influence on oculomotor function by performing electro-oculography or video-oculography to register eye movements. The variations in the eye-movement pattern associated with cervical rotation were suggested as a pathognomonic marker of proprioceptive cervical vertigo. Phillipszoon and $\operatorname{Bos}^{58}$ reported in 1963 that neck torsion nystagmus appears in cervical vertigo. Their test of cervical rotation, actually a test of the cervicoocular reflex, consists of rotating the body about the earth's vertical axis, while keeping the head still in space, and evaluating for nystagmus. This procedure has not been widely adopted, perhaps because of the requirement for a mechanism to rotate the body under the head. The cervico-ocular reflex also appears in other conditions, such as bilateral vestibular loss ${ }^{8,9}$ thus even if it were sufficiently sensitive, the finding of a cervico-ocular reflex could not be not a specific test for cervical vertigo. Furthermore, despite the recent wide availability of very sensitive video-Frenzel goggle testing that can detect small amounts of nystagmus with the head turned to either side at the bedside, this procedure appears to be insensitive and nonspecific (our unpublished observations).

A more-complicated procedure was described by Tjell and associates $^{59}$ in a 1998 study. They reported that the gain of the smooth-pursuit eye-movement test decreased significantly with 45-degree cervical rotation in patients with whiplash, as compared with patients with unspecified central vertigo, Ménière's disease, and normal controls. More recently, Treleaven et al in 2005 again noted that this test may be sensitive to whiplash, but with or without dizziness. ${ }^{60}$ Smooth pursuit is a complex multiple input system that is vulnerable to cognitive variables, age, and sedation. Neck pain and secondary gain, both disruptive of cognition, would also seem highly likely to influence performance of smooth pursuit. For these reasons, due to an intrinsic issue with specificity, it seems unlikely that any smooth-pursuit test could be of general utility for the diagnosis of cervical vertigo.

Kalberg and Magnusson described an asymmetry in the optokinetic afternystagmus in patients with asymmetric muscular activation similar to cervical vertigo. ${ }^{61}$ Optokinetic afternystagmus is difficult to obtain in humans and is generally of small velocity even in normal subjects. ${ }^{62}$ This makes it unlikely that this test could be sufficiently sensitive to be useful in cervical vertigo.

Several authors have reported that postural stability is especially reduced in patients with cervical injuries such as whiplash $^{63-66}$ This is a logical conjecture as injuries to the neck might disturb the computationally difficult adjustment between the two coordinate systems of the head and body. ${ }^{67}$ Impairment of postural stability, like smooth pursuit, is nonspecific and vulnerable to cognitive variables that might occur in situations where there is secondary gain; nevertheless, this approach is worth further development.

As there is no specific test to rule in cervical vertigo, vestibular laboratory tests are commonly performed to rule out other causes of dizziness and vertigo. Vestibular alterations represent the most common cause of vertigo in clinical practice. The following tests are the most relevant:

- Electronystagmography or videonystagmography: Evaluate the oculomotor and vestibular functions and explore the unilateral vestibular ocular reflex through caloric tests (localizing value)

- Rotatory chair testing: Evaluates the functionality of the vestibular system bilaterally

- Posturography: Evaluates the participation and integration of the sensory systems in balance and the effect of their deprivation (visual, proprioceptive, vestibular)

In most cases, videonystagmography has enough sensitivity to detect intrinsic vestibular disorders. Rotatory chair testing is often useful as an adjunct, as it should be confirmatory in vestibular disorders. Dynamic posturography can also be useful, especially in patients with persistent symptoms of nonspecific origin, in malingerers, in cases of psychiatric vertigo, or in patients with lawsuits where there is a higher possibility of behavior driven by hope of secondary gain. ${ }^{68}$

Imaging tests such as computed tomography (CT) or magnetic resonance imaging (MRI) allow one to detect anatomical alterations, malformations (e.g., Chiari malformation), expansive lesions (e.g., cerebellopontine angle tumor), traumatic alterations (e.g., brainstem contusion, spinal cord contusion, discopathies), or degenerative conditions such as cervical stenosis.

The use of MR angiography or CT angiography is especially useful to identify vascular defects that may present with vertigo in case of a cervical arterial compressive pathology (e. g., bow-hunter syndrome), although the dynamic sequences necessary to recognize a rotational cervical compression of the vertebral arteries are difficult from a technical standpoint (-Fig. 3). The most reliable and essential test to identify the 


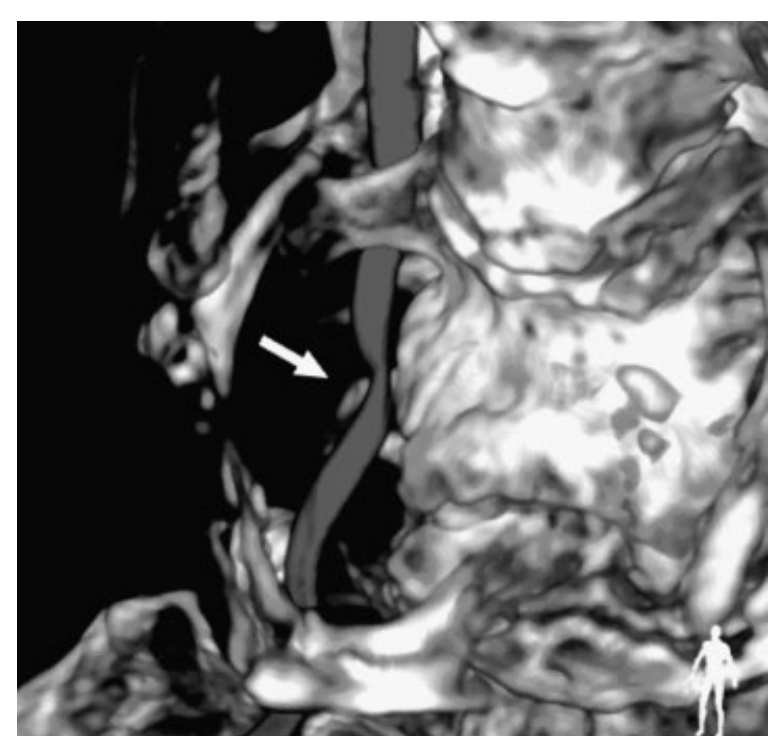

Fig. 3 Computed tomography angiography with three-dimensional reconstruction of neck arteries. An extrinsic compression of the right vertebral artery can be seen at the V2 level (arrow) due to a spur (osteophyte) in a case of bow-hunter syndrome.

exact area of mechanical vertebral compression and to make a surgical decision is digital subtraction angiography (DSA) of the supra-aortic vessels, with head rotation (rotation and extension). Nevertheless, since DSA is an invasive method requiring considerable iodine-containing contrast, it should be reserved for situations where suspicion is high, rather than as a screening test. We have also encountered some situations where radiologists are reluctant to perform tests such as DSA with head turning, in which the explicit goal is to document an iatrogenic occlusion of a vertebral artery.

A Doppler vascular test of the posterior blood flow with cervical rotation in search of decreased flow on posterior localization or posterior cerebral arteries is quick, noninvasive, and can be reliable. The Doppler test also allows one to identify subclavian steal syndrome, another cause of vertebrobasilar insufficiency. With Doppler, Sterk et al reported low values of vertebral artery velocity in $41 \%$ of the patients with tinnitus, vertigo, or degenerative cervical pathology. ${ }^{69}$ Machaly and associates also reported significantly lower blood flow as well as a higher prevalence of degenerative osteoarticular changes in patients with vertigo (71\%) compared with those without vertigo (33\%). ${ }^{70} \mathrm{~A}$ meta-analysis of nine studies concluded that vertebral artery blood flow is compromised with full contralateral rotation in healthy individuals. ${ }^{71}$ As discussed previously, it is highly unlikely that any significant proportion of cervical vertigo is due to circulatory disturbances. In other words, as vertebral Doppler abnormalities and cervical spondylosis are so common, tests of circulation and arthritis in the neck are likely to be highly nonspecific in the cervical vertigo population. Nevertheless, there may be subtle or subclinical forms of compressive vertebrobasilar insufficiency that have a discrete impact on the distal arteries (cochleovestibular). This finding is of particular concern in patients or older persons who are more likely to suffer from vascular pathology, such as atherosclerosis. It is also a potentially remediable cause.

To summarize, we presently do not have a method to rule in cervical vertigo. In particular, there are presently no special tests that can diagnose cervical vertigo. Vestibular laboratory tests serve mainly to exclude alternative innerear disorders as an alternative cause of vertigo. Imaging studies are useful to detect structural injury to the neck, which raises the probability of cervical vertigo, but they do not definitively rule it in or out. This leaves the clinician with a group of patients who might have cervical vertigo, but without a way to prove or exclude the diagnosis. Thus, the diagnostic situation for cervical vertigo resembles that of other clinical diagnoses in the dizzy population such as migraine-associated vertigo or chronic subjective vertigo, which also lack laboratory tests to rule them in or out.

\section{Treatment}

Ideally, the treatment should be clearly specified according to the condition that is causing the vertigo.

Recognizing vertigo in rotational vertebral arterial syndrome, a rare entity, is important for prognosis and therapy. When the exact area of the arterial compression is identified through appropriate tests such as DSA, decompressive surgery should be the chosen treatment. Due to the rareness of the syndrome, information about the treatment is based on isolated cases. So far, $\sim 43$ patients with this pathology undergoing surgery have been documented, and an algorithm for the management of this entity that ranges from decompression or surgical fusion to the application of a stent or conservative treatment (anticoagulant) has been published. $^{72}$

Lu et al presented a series of cases of nine patients that suffered from rotational vertebral arterial syndrome and concluded that the most common cause of arterial compression was an osteophyte (56\%), and the most frequent location was at the level of the first vertebra, C1 $(44 \%)^{73}$ (-Fig. 2). Surgical decompression was associated with the complete disappearance of the symptoms, although one patient (11\%) showed postsurgical complications (cervical instability).

Regarding the treatment of proprioceptive cervical vertigo, in which pain and imbalance or vertigo are the limiting symptoms, the quality of published studies in the current literature is poor. Most of these studies are neither controlled nor randomized. When the diagnosis is correct and other more common causes of vertigo are dismissed (e.g., vestibular pathology, central vascular pathology, etc.), manual physical therapy to reduce muscle contracture and muscle pain may be useful. Furman and Cass $^{25}$ suggested that vestibular rehabilitation associated with manual therapy could improve the patient's symptoms completely and rapidly. Along the same line, in two controlled studies, ${ }^{2,24}$ it was concluded that physical therapy is effective in treating patients with vertigo and cervicogenic dizziness. In the course of this treatment, the patient performs exercises of visual stability while the trunk is rotated, using glasses for foveal vision. In other words, 
cervico-ocular reflex exercises. Another physical therapy treatment currently being evaluated is passive joint mobilization through a manual therapy procedure called sustained natural apophyseal glides (SNAGs). ${ }^{2}$ The stated objective of some forms of physical therapy is to promote kinesthetic feedback, ${ }^{74}$ but such procedures might also work through reducing pain, spasm, or simply provide time for healing. Although it seems reasonable that joint manipulation could reduce pain and spasm, it is hard to understand how joint mobilization could increase proprioception.

Postwhiplash vertigo can combine several mechanisms. At the ear level, the otolith system is prone to suffer inertial damage, ${ }^{75}$ which can generate nonspecific dizziness and gait instability. Moreover, and as it was mentioned earlier, BPPV is by far the most common otologic disorder. If the right positional tests are employed, both entities must be correctly dismissed because the last is amenable to successful and inexpensive treatment by positional maneuvers. ${ }^{76}$

If other causes of vertigo have been already dismissed, the treatment for vertigo after whiplash injury affecting cervical soft tissues (ligaments, tendons, and muscles) usually consists of pharmacological treatment with analgesics and slow mobilization in its acute phase. There is agreement on discouraging the prolonged use of the cervical collar ${ }^{77}$ although as a general rule, avoidance of neck movement also reduces cervical vertigo.

Local heat may improve the stiffness and reduce the uneasiness during active mobilization. Using benzodiazepines for a short period can be useful. ${ }^{78}$ Antidepressants may improve emotional symptoms. Recovery is usually within 6 months; with 73 to $92 \%$ of patients able to return to work. $^{42} \mathrm{~A}$ program including physical training and balance can be as helpful as it is for other forms of vertigo. Intense cervical pain or focal neurologic symptoms should alert the clinician to the possibility of more severe complications (for instance, an unstable cervical spine or dissection).

\section{An Alternative Hypothesis for Some Forms of Cervical Vertigo}

As diagnosing cervicogenic vertigo includes the simultaneous presence of cervical pain, vertigo, aggravation with movement-and in the absence of alternative diagnoses-we briefly postulate the following hypothesis that derives from our daily clinical observation which could explain the clinical picture in a group of these patients.

Vertigo, dizziness, or imbalance associated with migraine is among the most prevalent entities. Migraine-associated vertigo represents the second most common cause of vertigo after BPPV. ${ }^{79,80}$ Vertigo occurs in 25 to $35 \%$ of nonselected migraine patients. ${ }^{81,82}$ Vertigo episodes may happen not only during the attacks, but also during the intervals free of migraine $^{82}$ (in between crises of pain). Kuryzky et al found that migraine patients had 2.5 times more vertigo attacks in periods free of pain with respect to a group of controls who did not suffer from migraines. ${ }^{83}$ The estimated prevalence of migraine-associated vertigo in the general population was $\sim 0.98 \%{ }^{84}$
On the other hand, cervical pain and stiffness is a typical finding of migraine. It has been reported that neck pain is statistically more often associated with migraine than nausea. ${ }^{85}$ Some reports show that cervical pain and stiffness may be premonitory (up to $35 \%$ of crisis), may trigger, and/or be additional to an episode of migraine. ${ }^{85-87}$ In a survey of a group of teenagers, $63 \%$ of the group of migraine patients reported cervical and shoulder pain. These cases are twice or three times more common than in patients who do not suffer from migraine. ${ }^{88}$

Accordingly, migraine-associated vertigo (35\%) with cervical pain or stiffness related to migraine (35-63\%), and the symptomatic definition of cervical vertigo overlap. They could constitute a new entity called "migraine-associated cervicogenic vertigo." Likewise, the sensory amplification produced by the migraine crisis could explain phonophobia, photophobia, and a related cervical vertigo symptom: head-movement intolerance. Up to $70 \%$ of episodes of migraine include cervical pain. ${ }^{86}$ The risk of triggering migraine is increased by cervical tension. ${ }^{89}$

A possible way to relate both entities in a bidirectional way would be the cervical trigeminal vestibular path. ${ }^{90,91}$ The reciprocal connections between the vestibular nuclei and the trigeminal nucleus caudalis can provide a mechanism in which the vestibular signals would influence the vascular trigeminal paths, widely related to the processing of the vestibular and trigeminal information during the migraine attacks. A similar activation of the cervical trigeminal path has been proposed and the results were allodynia and cervical hyperalgesia. ${ }^{91}$ Central sensitivity is another proposed mechanism to justify migraine symptoms.

This hypothesis of migraine-associated cervicogenic vertigo requires performing a specific study to be confirmed, which could clarify the dissociation observed between patients with cervical pain, but no dizziness or vertigo, and patients with dizziness and cervical pain. Even in the absence of structural abnormalities, migraine could be the difference.

\section{Conclusions}

Some entities that were previously defined as cervical vertigo have survived the test of time, such as vertigo in rotational vertebral arterial syndrome, proprioceptive cervicogenic vertigo, and its relative, posttraumatic cervical vertigo. Each has a different clinical picture, pathophysiological mechanism, prognosis, and treatment. Despite advances in laboratory tests and the well-documented connections between the cervical pathways and the balance system, the variant proprioceptive cervicogenic vertigo is still waiting for an objective diagnostic test. Rotational vertebral arterial syndrome presents a typical and known picture; although it is thought to be extremely rare, some vascular functional studies indicate that it could exist in a partial or subclinical form which is possibly being underestimated. Laboratory tests can be used either to dismiss other causes of vertigo or to identify the vascular involvement in cases of rotational vertebral arterial syndrome; in this event, treatment is individualized. We postulate that migraine could be a link that has not been 
previously identified to explain the clinical picture in some groups of these patients.

Even though it is presently impossible to measure the true extent of proprioceptive cervicogenic vertigo, manual and vestibular physical therapy seem to be the most reasonable therapeutic strategies.

\section{References}

1 Schappert SM. National Ambulatory Medical Care Survey: 1989 summary. Vital Health Stat 13 1992;(110):1-80

2 Reid SA, Rivett DA. Manual therapy treatment of cervicogenic dizziness: a systematic review. Man Ther 2005;10(1):4-13

3 Cherchi M. Infrequent causes of disequilibrium in the adult. Otolaryngol Clin North Am 2011;44(2):405-414, ixix

4 Somefun OA, Giwa OS, Bamgboye BA, Okeke-Igbokwe II, Azeez AA. Vestibular disorders among adults in a tertiary hospital in Lagos, Nigeria. Eur Arch Otorhinolaryngol 2010;267(10):1515-1521

5 Colledge NR, Barr-Hamilton RM, Lewis SJ, Sellar RJ, Wilson JA. Evaluation of investigations to diagnose the cause of dizziness in elderly people: a community based controlled study. BMJ 1996;313(7060):788-792

6 Brandt T. Cervical vertigo-reality or fiction? Audiol Neurootol 1996;1(4):187-196

7 Cullen KE, Roy JE. Signal processing in the vestibular system during active versus passive head movements. J Neurophysiol 2004;91 (5):1919-1933

8 Kelders WP, Kleinrensink GJ, van der Geest JN, Feenstra L, de Zeeuw $\mathrm{CI}$, Frens MA. Compensatory increase of the cervico-ocular reflex with age in healthy humans. J Physiol 2003;553(Pt 1): 311-317

9 Huygen PL, Verhagen WI, Nicolasen MG. Cervico-ocular reflex enhancement in labyrinthine-defective and normal subjects. Exp Brain Res 1991;87(2):457-464

10 Brandt T, Büchele W, Arnold F. Arthrokinetic nystagmus and egomotion sensation. Exp Brain Res 1977;30(2-3):331-338

11 Bles W, de Jong JM, de Wit G. Somatosensory compensation for loss of labyrinthine function. Acta Otolaryngol 1984;97(3-4):213-221

12 de Jong PT, de Jong JM, Cohen B, Jongkees LB. Ataxia and nystagmus induced by injection of local anesthetics in the Neck. Ann Neurol 1977;1(3):240-246

13 Dumas G, Perrin P, Schmerber S. Nystagmus induced by high frequency vibrations of the skull in total unilateral peripheral vestibular lesions. Acta Otolaryngol 2008;128(3):255-262

14 Koskimies K, Sutinen P, Aalto H, et al. Postural stability, neck proprioception and tension neck. Acta Otolaryngol Suppl 1997; 529:95-97

15 Dieterich M, Pöllmann W, Pfaffenrath V. Cervicogenic headache: electronystagmography, perception of verticality and posturography in patients before and after C2-blockade. Cephalalgia 1993; 13(4):285-288

16 Blouin J, Okada T, Wolsley C, Bronstein A. Encoding target-trunk relative position: cervical versus vestibular contribution. Exp Brain Res 1998;122(1):101-107

17 Popov KE, Lekhel H, Faldon M, Bronstein AM, Gresty MA. Visual and oculomotor responses induced by neck vibration in normal subjects and labyrinthine-defective patients. Exp Brain Res 1999;128(3):343-352

18 Ivanenko YP, Talis VL, Kazennikov OV. Support stability influences postural responses to muscle vibration in humans. Eur J Neurosci 1999;11(2):647-654

19 Padoan S, Karlberg M, Fransson PA, Magnusson M. Passive sustained turning of the head induces asymmetric gain of the vestibulo-ocular reflex in healthy subjects. Acta Otolaryngol 1998;118(6):778-782
20 Wapner S, Werner H, Morant RB. Experiments on sensory-tonic field theory of perception. III. Effect of body rotation on the visual perception of verticality. J Exp Psychol 1951;42(5):351-357

21 Brandt T. Vertigo: Its Multisensory Syndromes. 2nd ed. London: Springer; 1999

22 Wing LW, Hargrave-Wilson W. Cervical vertigo. Aust N Z J Surg 1974;44(3):275-277

23 Galm R, Rittmeister M, Schmitt E. Vertigo in patients with cervical spine dysfunction. Eur Spine J 1998;7(1):55-58

24 Karlberg M, Magnusson M, Malmström EM, Melander A, Moritz U. Postural and symptomatic improvement after physiotherapy in patients with dizziness of suspected cervical origin. Arch Phys Med Rehabil 1996;77(9):874-882

25 Furman JM, Cass SP. Balance disorders: a case-study approach. Philadelphia: F. A. Davis; 1996

26 Yahia A, Ghroubi S, Jribi S, et al. Chronic neck pain and vertigo: Is a true balance disorder present? Ann Phys Rehabil Med 2009;52(78):556-567

27 Wrisley DM, Sparto PJ, Whitney SL, Furman JM. Cervicogenic dizziness: a review of diagnosis and treatment. J Orthop Sports Phys Ther 2000;30(12):755-766

28 Heikkila H. Cervical Vertigo. In: Boyling J, Jull G, Twomey P, eds. Grieve's modern manual therapy: the vertebral column. 3rd ed. Edinburgh: Churchill Livingstone; 2004:233-242

29 Barré JA. Sur un syndrome sympathique cervical postérieur et sa cause frequente, l'arthrite cervicale. Paris: Revue Neurologique; 1926;1:1246-1248

30 Sadoshima S, Heistad DD. Regional cerebral blood flow during hypotension in normotensive and stroke-prone spontaneously hypertensive rats: effect of sympathetic denervation. Stroke 1983;14(4):575-579

31 Mueller SM, Heistad DD, Marcus ML. Total and regional cerebral blood flow during hypotension, hypertension, and hypocapnia. Effect of sympathetic denervation in dogs. Circ Res 1977;41 (3):350-356

32 Foster CA, Jabbour P. Barré-Lieou syndrome and the problem of the obsolete eponym. J Laryngol Otol 2007;121(7):680-683

33 Ryan GM, Cope S. Cervical vertigo. Lancet 1955;269(6905): $1355-1358$

34 Dix MR, Hallpike CS. The pathology symptomatology and diagnosis of certain common disorders of the vestibular system. Proc $\mathrm{R}$ Soc Med 1952;45(6):341-354

35 Brown JJ. Cervical contributions to balance: cervical vertigo. In: Berthoz A, Vidal PP, Graf W, eds., The Head Neck Sensory Motor System. New York, NY: Oxford University Press; 1992: 644-647

36 Morinaka S. Musculoskeletal diseases as a causal factor of cervical vertigo. Auris Nasus Larynx 2009;36(6):649-654

37 Strupp M, Arbusow V, Borges Pereira C, Dieterich M, Brandt T. Subjective straight-ahead during neck muscle vibration: effects of ageing. Neuroreport 1999;10(15):3191-3194

38 Schweigart G, Chien RD, Mergner T. Neck proprioception compensates for age-related deterioration of vestibular self-motion perception. Exp Brain Res 2002;147(1):89-97

39 Karnath HO, Reich E, Rorden C, Fetter M, Driver J. The perception of body orientation after neck-proprioceptive stimulation. Effects of time and of visual cueing. Exp Brain Res 2002;143 (3):350-358

40 Oosterveld WJ, Kortschot HW, Kingma GG, de Jong HA, Saatci MR. Electronystagmographic findings following cervical whiplash injuries. Acta Otolaryngol 1991;111(2):201-205

41 Skovron ML. Epidemiology of Whiplash. In: Szpalski M, Gunzburg $\mathrm{R}$, eds., Whiplash Injuries: Current Concepts in Preventions, Diagnosis, and Treatment of the Cervical Whiplash Syndrome. Philadelphia, PA: Lippincott-Raven; 1998:61-67

42 Pearce JM. A critical appraisal of the chronic whiplash syndrome. J Neurol Neurosurg Psychiatry 1999;66(3):273-276 
43 Rubin AM, Woolley SM, Dailey VM, Goebel JA. Postural stability following mild head or whiplash injuries. Am J Otol 1995;16 (2):216-221

44 Pang LQ. The otological aspects of whiplash injuries. Laryngoscope 1971;81(9):1381-1387

45 Toglia JU. Acute flexion-extension injury of the neck. Electronystagmographic study of 309 patients. Neurology 1976;26(9): 808-814

46 Endo K, Ichimaru K, Komagata M, Yamamoto K. Cervical vertigo and dizziness after whiplash injury. Eur Spine J 2006;15(6): 886-890

47 Ribbons T, Bell S. Neck pain and minor trauma: normal radiographs do not always exclude serious pathology. Emerg Med J 2008;25(9):609-610

48 Sorensen BF. Bow hunter's stroke. Neurosurgery 1978;2(3): 259-261

49 Toole JF, Tucker SH. Influence of head position upon cerebral circulation. Studies on blood flow in cadavers. Arch Neurol 1960;2:616-623

50 Grad A, Baloh RW. Vertigo of vascular origin. Clinical and electronystagmographic features in 84 cases. Arch Neurol 1989;46 (3):281-284

51 Takahashi I, Kaneko S, Asaoka K, Harada T. [Angiographic examination of the vertebral artery at the atlantoxial joint during head rotation]. No Shinkei Geka 1994;22(8):749-753

52 Choi KD, Shin HY, Kim JS, et al. Rotational vertebral artery syndrome: oculographic analysis of nystagmus. Neurology 2005; 65(8):1287-1290

53 Noh Y, Kwon OK, Kim HJ, Kim JS. Rotational vertebral artery syndrome due to compression of nondominant vertebral artery terminating in posterior inferior cerebellar artery. J Neurol 2011;258(10):1775-1780

54 Borg-Stein J, Rauch SD, Krabak B. Evaluation and management of cervicogenic dizziness. Critical Reviews in Physical Medicine and Rehabilitation 2001;13(4):255-264

55 Hohl M. Soft-tissue injuries of the neck in automobile accidents. Factors influencing prognosis. J Bone Joint Surg Am 1974;56(8): $1675-1682$

56 Maione A. Migraine-related vertigo: diagnostic criteria and prophylactic treatment. Laryngoscope 2006;116(10):1782-1786

57 Rahko T. The test and treatment methods of benign paroxysmal positional vertigo and an addition to the management of vertigo due to the superior vestibular canal (BPPV-SC). Clin Otolaryngol Allied Sci 2002;27(5):392-395

58 Philipszoon AJ, Bos JH. Neck torsion nystagmus. Pract Otorhinolaryngol (Basel) 1963;25:339-344

59 Tjell C, Rosenhall U. Smooth pursuit neck torsion test: a specific test for cervical dizziness. Am J Otol 1998;19(1):76-81

60 Treleaven J, Jull G, LowChoy N. Smooth pursuit neck torsion test in whiplash-associated disorders: relationship to self-reports of neck pain and disability, dizziness and anxiety. J Rehabil Med 2005;37 (4):219-223

61 Karlberg M, Magnusson M. Asymmetric optokinetic after-nystagmus induced by active or passive sustained head rotations. Acta Otolaryngol 1996;116(5):647-651

62 Tijssen MA, Straathof CS, Hain TC, Zee DS. Optokinetic afternystagmus in humans: normal values of amplitude, time constant, and asymmetry. Ann Otol Rhinol Laryngol 1989;98(9):741-746

63 Nacci A, Ferrazzi M, Berrettini S, et al. Vestibular and stabilometric findings in whiplash injury and minor head trauma. Acta Otorhinolaryngol Ital 2011;31(6):378-389

64 Mallinson AI, Longridge NS. Dizziness from whiplash and head injury: differences between whiplash and head injury. Am J Otol 1998;19(6):814-818

65 Kogler A, Lindfors J, Odkvist LM, Ledin T. Postural stability using different neck positions in normal subjects and patients with neck trauma. Acta Otolaryngol 2000;120(2):151-155
66 Yu LJ, Stokell R, Treleaven J. The effect of neck torsion on postural stability in subjects with persistent whiplash. Man Ther 2011;16 (4):339-343

67 Nashner LM, Wolfson P. Influence of head position and proprioceptive cues on short latency postural reflexes evoked by galvanic stimulation of the human labyrinth. Brain Res 1974;67(2): 255-268

68 Cevette MJ, Puetz B, Marion MS, Wertz ML, Muenter MD. Aphysiologic performance on dynamic posturography. [see comments]Otolaryngol Head Neck Surg 1995;112(6):676688

69 Strek P, Reroń E, Maga P, Modrzejewski M, Szybist N. A possible correlation between vertebral artery insufficiency and degenerative changes in the cervical spine. Eur Arch Otorhinolaryngol 1998;255(9):437-440

70 Machaly SA, Senna MK, Sadek AG. Vertigo is associated with advanced degenerative changes in patients with cervical spondylosis. Clin Rheumatol 2011;30(12):1527-1534

71 Mitchell J. Vertebral artery blood flow velocity changes associated with cervical spine rotation: a meta-analysis of the evidence with implications for professional practice. J Manual Manip Ther 2009;17(1):46-57

72 Cornelius JF, George B, N'dri Oka D, Spiriev T, Steiger HJ, Hänggi D. Bow-hunter's syndrome caused by dynamic vertebral artery stenosis at the cranio-cervical junction-a management algorithm based on a systematic review and a clinical series. Neurosurg Rev 2012;35(1):127-135, discussion 135

73 Lu DC, Zador Z, Mummaneni PV, Lawton MT. Rotational vertebral artery occlusion-series of 9 cases. Neurosurgery 2010;67 (4):1066-1072, discussion 1072

74 Clendaniel R. Cervical vertigo. In: Herdman S, ed., Vestibular Rehabilitation. 2nd ed. Philadelphia, PA: Davis; 2000: 494-509

75 Lee JD, Park MK, Lee BD, Park JY, Lee TK, Sung KB. Otolith function in patients with head trauma. Eur Arch Otorhinolaryngol 2011;268(10):1427-1430

76 Hilton M, Pinder D. The Epley (canalith repositioning) manoeuvre for benign paroxysmal positional vertigo. Cochrane Database Syst Rev 2004;(2):CD003162

77 Jansen GB, Edlund C, Grane P, et al; Swedish Society of Medicine; Whiplash Commission Medical Task Force. Whiplash injuries: diagnosis and early management. Eur Spine J 2008;17(Suppl 3): S355-S417

78 Peloso P, Gross A, Haines T, Trinh K, Goldsmith CH, Burnie S; Cervical Overview Group. Medicinal and injection therapies for mechanical neck disorders. Cochrane Database Syst Rev 2007;(3): CD000319

79 Neuhauser H, Leopold M, von Brevern M, Arnold G, Lempert T. The interrelations of migraine, vertigo, and migrainous vertigo. Neurology 2001;56(4):436-441

80 Neuhauser HK, Lempert T. Vertigo: epidemiologic aspects. Semin Neurol 2009;29(5):473-481

81 Selby G, Lance JW. Observations on 500 cases of migraine and allied vascular headache. J Neurol Neurosurg Psychiatry 1960;23:23-32

82 Kayan A, Hood JD. Neuro-otological manifestations of migraine. Brain 1984;107(Pt 4):1123-1142

83 Kuritzky A, Ziegler DK, Hassanein R. Vertigo, motion sickness and migraine. Headache 1981;21(5):227-231

84 Lempert T, Neuhauser H. Epidemiology and clinical aspect of migraine-associated dizziness (abstract 173). J Vest Res 2004; $14: 180$

85 Calhoun AH, Ford S, Millen C, Finkel AG, Truong Y, Nie Y. The prevalence of neck pain in migraine. Headache 2010;50(8):12731277

86 Kelman L. Migraine pain location: a tertiary care study of 1283 migraineurs. Headache 2005;45(8):1038-1047 
87 Schoonman GG, Evers DJ, Terwindt GM, van Dijk JG, Ferrari MD. The prevalence of premonitory symptoms in migraine: a questionnaire study in 461 patients. Cephalalgia 2006;26(10):1209-1213

88 Blaschek A, Milde-Busch A, Straube A, et al. Self-reported muscle pain in adolescents with migraine and tension-type headache. Cephalalgia 2012;32(3):241-249

89 Wöber C, Brannath W, Schmidt K, et al; PAMINA Study Group. Prospective analysis of factors related to migraine attacks: the PAMINA study. Cephalalgia 2007;27(4):304-314
90 Fernández-de-Las-Peñas C, Cuadrado ML, Arendt-Nielsen L, Pareja JA. Side-to-side differences in pressure pain thresholds and pericranial muscle tenderness in strictly unilateral migraine. Eur J Neurol 2008;15(2):162-168

91 Buisseret-Delmas C, Compoint C, Delfini C, Buisseret P. Organisation of reciprocal connections between trigeminal and vestibular nuclei in the rat. J Comp Neurol 1999;409(1):153-168 